Real Analysis Exchange

Vol. 24(2), 1998/9, pp. 579-588

Frank S. Cater, Department of Mathematics, Portland State University, Portland, Oregon 97207, USA

\title{
VARIATIONS ON A THEOREM ON RINGS OF CONTINUOUS FUNCTIONS
}

\begin{abstract}
We find variations of the classical theorem that any nonzero real ring homomorphism, $u$, of $C(X)$, for a compact Hausdorff space, $X$, is fixed. We let $X$ be a locally compact Hausdorff space and we let $u$ be defined on certain subrings of $C(X)>$ We also vary the hypothesis on $u$ in other ways.
\end{abstract}

\section{Introduction}

For a topological space $X$, let $C(X)$ denote the ring of all continuous real valued functions on $X$, and let $C^{*}(X)$ denote the subring of all bounded functions in $C(X)$. If $X$ is locally compact Hausdorff, let $C^{* *}(X)$ denote the family $\{f \in C(X): f-1$ vanishes at infinity $\}$. Hence $C^{* *}(X) \subset C^{*}(X)$. (Note: we say that $g$ vanishes at infinity [GJ, 7FG] if the set $\{x \in X$ : $|g(x)| \geq 1 / n\}$ is compact for all integers $n>0$. We say that $p$ is the limit of $g$ at infinity if $g-p$ vanishes at infinity.)

We say that a real ring homomorphism $u$ of a subring $S$ of $C(X)$ is fixed [GJ, 10.5], if for some $x_{0} \in X, f\left(x_{0}\right)=u(f)$ for all $f \in S$. A completely regular Hausdorff space $X$ is said to be realcompact [GJ, 10.5], if every nonzero real ring homomorphism on $C(X)$ is fixed. Perhaps the best known result on realcompact spaces is $[\mathrm{K}, 7 \mathrm{~S}]$, [GJ, 8.2].

Proposition A. Every compact Hausdorff space $X$ is realcompact.

In Section 4 we will discover a proof of Proposition A that does not require the use of ideals. Unfortunately a locally compact Hausdorff space need not be realcompact. The standard counterexample [GJ, 5.12] is the space $X$ of

Key Words: $C(X), C^{*}(X)$, ring, vector space, ring homomorphism, linear functional, completely regular, compact, locally compact, realcompact, binary operation.

Mathematical Reviews subject classification: 26A15, 54C30, 54D30, 54P60.

Received by the editors March 3, 1998 
countable ordinal numbers under the order topology. If however we define a binary operation $*$ from $\mathbb{R}^{2}$ to $\mathbb{R}$ by

$$
a * b=a-a b
$$

we can achieve something that resembles realcompactness in a limited way, for locally compact spaces $X$. In Theorem 2.1 we let $u$ be a nonzero real function preserving $*$,

$$
u(f-f g)=u(f)-u(f) u(g),
$$

on a subring $S$ of $C(X)$ containing $C^{* *}(X)$ such that $u$ is nonconstant on $C^{* *}(X)$, and we prove that $u$ is fixed. Note that our theorem requires that $u$ be nonconstant on $C^{* *}(X)$, and that $u$ preserve only the one operation *, rather than the two, addition and multiplication. Moreover, note that the subring $S$ need not be $C(X)$ or $C^{*}(X)$, provided $C^{* *}(X) \subset S$.

In Theorem 3.1 we let $u$ be a nonzero real valued function on a subring $S$ of $C(Y)$ containing $C^{*}(Y)$, where $Y$ is any topological space. We let $u$ preserve * and prove that $u$ must be a ring homomorphism on $S$.

This differs from Theorem 2.1 in that we have all the bounded continuous functions at our disposal in $S$. Note there is no restriction on the space $Y$. If for example, $Y$ is discrete, then Theorem 3.1 is just a statement about real valued functions on the set $Y$. In Section 3 we also offer some applications to realcompact spaces.

In Section 4 we let $X$ be compact Hausdorff and regard $C(X)$ to be a real vector space. We let $L$ be a linear subspace of $C(X)$ containing 1 , and let $u$ be a nonzero real linear functional on $L$ with $u \geq 0$ and $u(1)=1$. In Theorem 4.1 we prove that under certain hypotheses $u$ must be fixed. We conclude with some examples arising from Theorem 4.1.

\section{The Locally Compact Hausdorff Case}

We begin with the following assertion.

Theorem 2.1. Let $X$ be a locally compact Hausdorff space, and let $S$ be a subring of $C(X)$ containing $C^{* *}(X)$. Let $u$ be a real valued function on $S$ that is not constant on $C^{* *}(X)$ such that

$$
u(f * g)=u(f) * u(g) \quad(f, g \in S) .
$$

Then $u$ is a real fixed ring homomorphism on $S$. Moreover, $u$ extends to a unique real ring homomorphism on $C(X)$. 
We will prove fairly easily that $u$ preserves multiplication. Most of the work will be in the remainder of the proof, where we will use the one-point compactification to establish that $u$ preserves addition.

Proof OF Theorem 2.1. We have by hypothesis $u(0)=u\left(0-0^{2}\right)=u(0)-$ $u(0)^{2}$ and hence $u(0)=0$. Also $0=u(0)=u\left(1-1^{2}\right)=u(1)-u(1)^{2} ;$ so $u(1)$ is either 0 or 1 . But $u(1)=0$ is impossible because otherwise

$$
u(1-f)=u(1-1 f)=u(1)-u(1) u(f)=0
$$

for any $f \in S$, and consequently $u$ would vanish on $S$ and $C^{* *}(X)$, contrary to hypothesis. Hence $u(1)=1$. Now for any $f \in S$

$$
u(1-f)=u(1-1 f)=u(1)-u(1) u(f)=1-u(f) .
$$

For any $f, g \in S$,

$$
\begin{aligned}
u(f g) & =u(f-f(1-g))=u(f)-u(f) u(1-g)= \\
& =u(f)-u(f)(1-u(g))=u(f) u(g) .
\end{aligned}
$$

For $f, g \in S$ we have established

(i) $u(f g)=u(f) u(g)$,

(ii) $u(1-f)=1-u(f)$.

Let $\{p\} \cup X$ be the one point compactification of $X$ where $p$ is the point at infinity. Let $D$ denote the family of all continuous functions $f$ from $\{p\} \cup X$ into $[0,1]$ with $f(p)=1$. Essentially $D \subset C^{* *}(X)$. Let $1-D$ denote $\{1-f: f \in$ $D\}$. Now $D \cup(1-D)$ is closed under multiplication $(f, g \in D \cup(1-D)$ implies $f g \in D \cup(1-D))$, and is closed under complementation $(f \in D \cup(1-D)$ implies $1-f \in D \cup(1-D))$. Moreover (i) and (ii) hold for functions in $D \cup(1-D)$.

Let $f \in D$. By (ii), $u(1-f)=1-u(f)$. But $f^{\frac{1}{2}} \in D$ and $(1-f)^{\frac{1}{2}} \in 1-D$. Then by (i), $u(f)=u\left(f^{\frac{1}{2}}\right)^{2} \geq 0$ and $u(1-f)=u\left((1-f)^{\frac{1}{2}}\right)^{2} \geq 0$. Clearly $u$ maps $D \cup(1-D)$ into $[0,1]$. It follows from [C, Theorem 1$]$ that there is an $x_{0} \in\{p\} \cup X$ such that $f\left(x_{0}\right)=u(f)$ for all $f \in D \cup(1-D)$. Our next task is to prove that $x_{0} \neq p$.

Let $h \in C^{* *}(X)$. Put $h(p)=1$. Let $k$ be a function in $D($ with $k(p)=1)$ that vanishes on the closed set $\{x \in X: h(x) \leq 1 / 2\}$ such that $k \leq 1 / h$ on the support of $k$. Then $h k \in D$ and $u(h k)=u(h) u(k)$. Now if $x_{0}=p$, then

$$
1=(h k)(p)=u(h k)=u(h) u(k) \quad \text { and } \quad 1=k(p)=u(k) .
$$


Hence $u(h)=1$, contrary to the hypothesis that $u$ is not constant on $C^{* *}(X)$. We have established that $x_{0} \in X$.

Let $f \in S$ with $f\left(x_{0}\right)>0$. Let $f_{1}$ be a function in $1-D$ such that $f_{1}$ vanishes on the closed set $\left\{x \in X: f(x) \leq f\left(x_{0}\right) / 2\right\}, f_{1}\left(x_{0}\right)>0$, and $f_{1} \leq 1 / f$ on the support of $f_{1}$. Then $f f_{1} \in 1-D$. So by (i),

$$
f\left(x_{0}\right) f_{1}\left(x_{0}\right)=\left(f f_{1}\right)\left(x_{0}\right)=u\left(f f_{1}\right)=u(f) u\left(f_{1}\right)=u(f) f_{1}\left(x_{0}\right),
$$

and (because $\left.f_{1}\left(x_{0}\right) \neq 0\right) u(f)=f\left(x_{0}\right)$. Let $f_{0} \in S$ with $f_{0}\left(x_{0}\right) \leq 0$. By applying (ii) and this argument to $1-f_{0}$ we see that

$$
1-f_{0}\left(x_{0}\right)=u\left(1-f_{0}\right)=1-u\left(f_{0}\right) \quad \text { and } \quad u\left(f_{0}\right)=f_{0}\left(x_{0}\right) .
$$

Thus $g\left(x_{0}\right)=u(g)$ for all $g \in S$. Hence $\left(g_{1}+g_{2}\right)=u\left(g_{1}\right)+u\left(g_{2}\right)$ for $g_{1} \in$ $S, g_{2} \in S$.

Now $u$ extends to an obvious ring homomorphism of $C(X)$ to $\mathbb{R}$. If $v$ is any other ring homomorphism of $C(X)$ to $\mathbb{R}$ that extends $u$, the argument in the preceding paragraph shows that $g\left(x_{0}\right)=v(g)$ for all $g \in C(X)$.

Corollary 2.2. Let $S$ and $X$ be as in Theorem 2.1. Let $W$ be any space, and let $U$ be a mapping from $S$ into $C(W)$ such that $U\left(C^{* *}(X)\right)(w)$ is not a singleton set for any $w \in W$, and $U(f * g)=U(f) * U(g) \quad(f, g \in S)$. Then there is a continuous function $t$ from $W$ into $X$ such that $U(f)(w)=$ $f(t(w)) \quad(f \in S, w \in W)$.

This is proved by an argument like the solution to [K, problem 7S, pp. 245-6].

A real valued mapping preserving operation $*$ on an arbitrary commutative ring with identity need not be a ring homomorphism. For example, consider the mapping on the ring of integers taking even integers to 0 and odd integers to 1 . This inspires the following corollary.

Corollary 2.3. Let $Y$ be a compact space and let $p \in Y$. Let $S$ be the family $\{f \in C(Y): f(p)$ is an integer $\}$. Let $u$ be a nonzero mapping from $S$ into $\mathbb{R}$ such that

$$
u(f * g)=u(f) * u(g) \quad(f, g \in S) .
$$

Then either $u$ is fixed, or $u(f)=0$ when $f(p)$ is even and $u(f)=1$ when $f(p)$ is odd.

Proof. First note that $C^{* *}(X) \subset S$. Our proof then proceeds just like the proof of Theorem 2.1 until (i) and (ii) are proved, $D$ and $1-D$ are defined, and $x_{0}$ is found. If $x_{0} \neq p$, the conclusion follows just as in the proof of Theorem 
2.1. It suffices then to let $x_{0}=p$. As before, $u(1)=1$ and $u(0)=0$. Now $u(-1)^{2}=u(1)=1$ by (i); so $u(-1)=1$ or -1 .

CASE 1. $u(-1)=-1$. Here $u(2)=u(1-(-1))=1-u(-1)=2$ by (ii). By (i), $u(-2)=u(-1) u(2)=-2$. So $u(3)=u(1-(-2))=1-u(-2)=3$. Similarly, $u(-3)=-3$ and $u(4)=4$. Clearly an induction argument will establish that $u(n)=n$ for any integer, positive, negative or zero.

Let $f \in S$ with $f(p)=1$. Let $f_{0} \in D$ such that $f_{0}$ vanishes on the closed set $\{x \in X: f(x) \leq 1 / 2\}$ and $f_{0} \leq 1 / f$ on the support of $f_{0}$. Then $f f_{0} \in D$ and $f_{0} \in D$; so by (i) $f(p)=1=\left(f f_{0}\right)(p)=u\left(f f_{0}\right)=u(f) u\left(f_{0}\right)=u(f)$. Now let $g \in S$ and $g(p)=n$ where $n$ is a nonzero integer. We have $n^{-1} g \in S$, $\left(n^{-1} g\right)(p)=1$, and by the preceding paragraph, $u\left(n^{-1} g\right)=1$. Finally, $u(g)=$ $u(n) u\left(n^{-1} g\right)=n$ by (i). If $h \in S$ and $h(p)=0$, then $1=u(1-h)=1-u(h)$ because $(1-h)(p)=1$. So $u(h)=0$. Thus in Case $1, u$ is fixed.

CASE 2. $u(-1)=1$. Here $u(2)=u(1-(-1))=1-u(-1)=0$. Thus if $f \in S$ and $f(p)$ is even, then $f / 2 \in S$ and by (i), $u(f)=u(2) u(f / 2)=0$. If $g(p)$ is odd, then $(1-g)(p)=1-g(p)$ is even and hence $0=u(1-g)=1-u(g)$ and $u(g)=1$.

Note that of all the mappings $u$ possible in Corollary 2.3, the two associated with the point $p$ have countable range and all the others have uncountable range. Now let $V$ be a locally compact Hausdorff, noncompact space and let $S_{V}$ denote the family $\{f \in C(V)$ : the limit of $f$ at infinity is an integer $\}$. Arguments similar to the solution to $[\mathrm{K}$, problem $7 \mathrm{~S}(\mathrm{e})$, p. 246] prove that the algebraic structure $\left(S_{V}, *\right)$ determines the space $V$ in the following sense. If $V_{1}$ and $V_{2}$ are such spaces and if $\left(S_{V_{1}}, *\right)$ and $\left(S_{V_{2}}, *\right)$ are isomorphic, then $V_{1}$ and $V_{2}$ are homomorphic. Of course the ring $S_{V}$ also determines $V$, but again the one operation $*$ suffices.

\section{$3 \quad$ Arbitrary Spaces and Realcompact Spaces}

We first establish the following assertion about arbitrary spaces.

Theorem 3.1. Let $Y$ be any topological space and let $S$ be any subring of $C(Y)$ that contains $C^{*}(Y)$. Let $u$ be a nonzero real valued function on $S$ such that $u(f * g)=u(f) * u(g) \quad(f, g \in S)$. Then $u$ is a real ring homomorphism on $S$.

We begin the proof with a lemma.

Lemma. Let $Y$ be any space and let $S$ be a subring of $C(Y)$ such that $C^{*}(Y) \subset$ $S$. Let $f \in S$. Then $\max (0, f) \in S$ and $\min (0, f) \in S$. 
Proof. Let $g=\min (1, \max (0, f))$. Then $g \in C^{*}(Y)$ and hence $g \in S$. Observe that $f g$ vanishes on the set $\{y \in Y: f(y) \leq 0\}$ and $f g$ coincides with $f$ on the set $\{y \in Y: f(y) \geq 1\}$. It follows that $f-f g=\min (0, f)+h$ where $h \in C^{*}(Y) \subset S$. But $f-f g \in S$; so $\min (0, f) \in S$. Finally, $\max (0, f)=$ $f-\min (0, f) \in S$.

Proof. [Proof of Theorem 3.1] Just as in the proof of Theorem 2.1 we prove that $u(0)=0, u(1)=1$ and prove that (i) and (ii) hold. We claim that $u(2) \neq 0$; for otherwise, $1=u(1)=u(2) u(1 / 2)=0$. But by $(\mathrm{i}), 1=u(1)=$ $u(-1)^{2}$ and $u(-1)=1$ or -1 . We claim that $u(-1) \neq 1$; for otherwise $u(2)=u(1-(-1))=1-u(-1)=0$ by (ii). Hence $u(-1)=-1$. If $g \in S$, then by (i), $u(-g)=u(-1) u(g)=-u(g)$.

In what follows, $k_{1}$ and $k_{2}$ are in $S$ and $k_{1} \geq 0$. Then $\left(1+k_{1}\right)^{-1} \in C^{*}(Y) \subset$ $S$ and $\left(1+k_{1}\right)^{-1}\left(-k_{2}\right) \in S$. By (i), (ii) and $u\left(-k_{2}\right)=-u\left(k_{2}\right)$,

$u\left(1+k_{1}-\left(1+k_{1}\right)\left(1+k_{1}\right)^{-1}\left(-k_{2}\right)\right)=u\left(1+k_{1}\right)-u\left(-k_{2}\right)=u\left(1+k_{1}\right)+u\left(k_{2}\right)$.

Moreover $u\left(1-\left(-k_{1}\right)\right)=1-u\left(-k_{1}\right)=1+u\left(k_{1}\right)$. Combining,

$$
u\left(1+k_{1}+k_{2}\right)=1+u\left(k_{1}\right)+u\left(k_{2}\right) .
$$

Again by (ii), $u\left(1+k_{1}+k_{2}\right)=1-u\left(-k_{1}-k_{2}\right)=1+u\left(k_{1}+k_{2}\right)$. Thus

$$
u\left(1+k_{1}+k_{2}\right)=1+u\left(k_{1}+k_{2}\right) .
$$

From (1) and (2) we obtain $1+u\left(k_{1}\right)+u\left(k_{2}\right)=1+u\left(k_{1}+k_{2}\right)$, or

$$
u\left(k_{1}+k_{2}\right)=u\left(k_{1}\right)+u\left(k_{2}\right) .
$$

Now let $f, g \in S$. Say $f_{+}=\max (0, f), g_{+}=\max (0, g), f_{-}=-\min (0, f)$, $g_{-}=-\min (0, g)$. Then $f_{+}, g_{+}, f_{-}, g_{-}$are all nonnegative, $f=f_{+}-f_{-}$, $g=g_{+}-g_{-}$, and by the Lemma, $f_{+}, g_{+}, f_{-}, g_{-}$are all in $S$. By the preceding paragraph,

$$
\begin{aligned}
u(f+g) & =u\left(f_{+}+g_{+}-f_{-}-g_{-}\right) \\
& =u\left(f_{+}+g_{+}\right)+u\left(-f_{-}-g_{-}\right) \\
& =u\left(f_{+}+g_{+}\right)-u\left(f_{-}+g_{-}\right) \\
& =u\left(f_{+}\right)+u\left(g_{+}\right)-u\left(f_{-}\right)-u\left(g_{-}\right) .
\end{aligned}
$$

Likewise

$$
\begin{aligned}
u(f)+u(g) & =u\left(f_{+}-f_{-}\right)+u\left(g_{+}-g_{-}\right) \\
& =u\left(f_{+}\right)+u\left(-f_{-}\right)+u\left(g_{+}\right)+u\left(g_{-}\right) \\
& =u\left(f_{+}\right)+u\left(g_{+}\right)-u\left(f_{-}\right)-u\left(-g_{-}\right) .
\end{aligned}
$$

By comparing, we obtain $u(f+g)=u(f)+u(g)$. 
This corollary is almost immediate.

Corollary 3.2. Let $Y$ be a realcompact space, let $u$ be a real valued function on $C(Y)$ with $u(1)=1$ satisfying $u(f * g)=u(f) * u(g) \quad(f, g \in C(Y))$. Then $u$ is a fixed ring homomorphism of $C(Y)$ into $\mathbb{R}$.

Proof. That $u$ is a ring homomorphism on $C(Y)$ follows from Theorem 3.1. Because $Y$ is realcompact, $u$ is fixed [GJ, 10.5].

Corollary 3.3. Let $X$ be realcompact. Let $W$ be any space, and let $U$ be a mapping from $C(X)$ into $C(W)$ such that $U(1)=1$ and

$$
U(f * g)=U(f) * U(g) \quad(f, g \in C(X)) .
$$

Then there is a continuous function $t$ from $W$ into $X$ such that

$$
U(f)(w)=f(t(w)) \quad(f \in C(X), w \in W) .
$$

The proof is similar to the solution of $[\mathrm{K}$, problem $7 \mathrm{~S}(\mathrm{e})$, p. 246$]$; so we leave it. We note the algebraic structure $(C(V), *)$ determines a realcompact space $V$ in the following way. If $V_{1}$ and $V_{2}$ are realcompact spaces and if $\left(C\left(V_{1}\right), *\right)$ and $\left(C\left(V_{2}\right), *\right)$ are isomorphic, then $V_{1}$ and $V_{2}$ are homomorphic. Of course the ring $C(V)$ also determines $V$, but again the one operation * suffices.

\section{Compact Hausdorff Spaces}

Here we prove the following theorem.

Theorem 4.1. Let $X$ be a compact Hausdorff space and regard $C(X)$ to be a real vector space. Let $L$ be a linear subspace of $C(X)$ containing 1 , and let $u$ be a nonzero real linear functional on $L$ with $u \geq 0$ and $u(1)=1$. Let

$$
K=\left\{f \in L: f^{2} \in L \text { and } u\left(f^{2}\right)=(u(f))^{2}\right\},
$$

and let $A$ be the smallest uniformly closed subalgebra of $C(X)$ containing $K$. Then there is an $x_{0} \in X$ such that $u(f)=f\left(x_{0}\right)$ for all $f \in L \cap A$. Moreover, if $K$ separates points in $X$, then $u(f)=f\left(x_{0}\right)$ for all $f \in L$.

Proof. Let $f_{1}, f_{2}, \ldots, f_{n}$ be finitely many functions in $K$. Let

$$
F=\left(f_{1}-u\left(f_{1}\right)\right)^{2}+\left(f_{2}-u\left(f_{2}\right)\right)^{2}+\ldots+\left(f_{n}-u\left(f_{n}\right)\right)^{2} .
$$


Now

$$
\begin{aligned}
u\left(\left(f_{i}-u\left(f_{i}\right)\right)^{2}\right) & =u\left(f_{i}^{2}-2 u\left(f_{i}\right) f_{i}+u\left(f_{i}\right)^{2}\right) \\
& =u\left(f_{i}\right)^{2}-2 u\left(f_{i}\right)^{2}+u\left(f_{i}\right)^{2}=0
\end{aligned}
$$

by the linearity of $u$, and it follows that $u(F)=0$. But $F \geq 0$ on $X$.

We claim that $F$ vanishes at some point in $X$. Assume not. By compactness, there is a number $d>0$ such that $F \geq d$ on $X$. Because $u \geq 0$, we have $u(F) \geq u(d)=d>0=u(F)$, which is impossible. So there is some point $x_{1} \in X$ with $F\left(x_{1}\right)=0$. But then $f_{1}\left(x_{1}\right)=u\left(f_{1}\right), f_{2}\left(x_{1}\right)=$ $u\left(f_{2}\right), \ldots, f_{n}\left(x_{1}\right)=u\left(f_{n}\right)$. It follows then that for each $f \in K$, there is a nonvoid closed set $X_{f} \subset X$ with $f(x)=u(f)$ for $x \in X_{f}$. The family $X_{f}$ $(f \in K)$ has the finite intersection property; so by compactness there is a point $x_{0} \in \cap_{f \in K} X_{f}$. Thus $f\left(x_{0}\right)=u(f)$ for all $f \in K$. It remains to prove that $g\left(x_{0}\right)=u(g)$ for all $g \in L \cap A$. (Observe that we now have a proof of Proposition A without ideals.)

Let $V=\left\{f \in L: f\left(x_{0}\right)=u(f)\right\}$. Then $K \subset V$ and indeed if $f \in K$ then $f \in V$ and $f^{2} \in V$. Moreover, if $f \in K$ and $a$ is a real number, then

$$
\begin{aligned}
u\left(a(f-u(f))^{2}\right) & =a u\left(f^{2}-2 u(f) f+u(f)^{2}\right) \\
& =a\left(u(f)^{2}-2 u(f)^{2}+u(f)^{2}\right)=0 .
\end{aligned}
$$

Select $g \in L \cap A$ and let $c$ be a number $>g\left(x_{0}\right)$. The set $P=\{x \in X$ : $g(x) \geq c\}$ is a (closed) compact subset of $X$ and $x_{0} \notin P$. Take $y \in P$. Then $g$ separates $x_{0}$ and $y$, and because $g \in A$ there exists a positive number $a$ and $f \in K$ such that $a\left(f(y)-f\left(x_{0}\right)\right)^{2}>g(y)-c$. Because $P$ is compact, there are finitely many positive numbers $a_{1}, a_{2}, \ldots, a_{n}$ and functions $f_{1}, f_{2}, \ldots, f_{n} \in K$ such that

$$
a_{1}\left(f_{1}-u\left(f_{1}\right)\right)^{2}+a_{2}\left(f_{2}-u\left(f_{2}\right)\right)^{2}+\ldots+a_{n}\left(f_{n}-u\left(f_{n}\right)\right)^{2}>g-c
$$

on $P$. Put

$$
G=c+\sum_{i=1}^{n} a_{i}\left(f_{i}-u\left(f_{i}\right)\right)^{2} \in V .
$$

Then $G \geq g$ on $X$ and $u(G)=c$. Because $u \geq 0$ we have $c=u(G) \geq u(g)$. But $c\left(>g\left(x_{0}\right)\right)$ was arbitrary; so $g\left(x_{0}\right) \geq u(g)$. By the same kind of argument $-g\left(x_{0}\right) \geq u(-g)=-u(g)$; so finally $g\left(x_{0}\right)=u(g)$.

If $K$ separates points in $X$, then $A=C(X)$ by the Stone-Weierstrass Theorem [GJ, (16.4)], and $g\left(x_{0}\right)=u(g)$ for all $g \in L$. 
(Observe that we used compactness on three occasions in this proof; once to show that $F$ vanishes at some point, once to obtain $x_{0}$, and finally to obtain the function $G$.)

We turn to Borel measures.

Corollary 4.2. Let $X$ be a compact Hausdorff space and let $m$ be a nonnegative regular Borel measure on $X$ with $m(X)=1$. Let

$$
K=\left\{f \in C(X):\left(\int f d m\right)^{2}=\int f^{2} d m\right\}
$$

and let $K$ separate points in $X$. Then there is a singleton subset of $X$ whose complement has measure zero.

We omit the proof. Note that if $x_{0}$ is a point satisfying $f\left(x_{0}\right)=\int f d m$ for $f \in C(X)$, and if $h \in C(X)$ such that $0 \leq h \leq 1$ on $X, h\left(x_{0}\right)=0$ and $h=1$ on a compact set $P$ not containing $x_{0}$, we see that $m(P)=0$.

Example 1. Let $L$ be the linear space spanned by the functions $1, \cos (n x)$ $(n=1,2,3, \ldots)$ on $[0, \pi]$. Let $u$ be a nonnegative linear functional on $L$ with $u(1)=1$. Let

$$
u(\cos (2 x))=2(u(\cos x))^{2}-1
$$

Then $u$ is fixed on $L$.

Proof. We have

$$
u(\cos (2 x))=u\left(2 \cos ^{2} x-1\right)=2 u\left(\cos ^{2} x\right)-1=2(u(\cos x))^{2}-1,
$$

and hence $u\left(\cos ^{2} x\right)=(u(\cos x))^{2}$. The rest follows from Theorem 4.1.

Example 2. Let $W$ be the compact square $[0,1] \times[0,1]$ in $\mathbb{R}^{2}$. Let $u$ be a nonnegative linear functional on $C(W)$ with $u(1)=1$. Let $g(x, y)=x$, $h(x, y)=y$ on $W$. Let $(u(g))^{2}=u\left(g^{2}\right),(u(h))^{2}=u\left(h^{2}\right)$. Then $u$ is fixed on $C(W)$.

We omit the proof.

Example 3. Let $u$ be a nonnegative linear functional on $C[0, \pi]$ with $u(1)=1$, $u\left(g^{2}\right)=(u(g))^{2}, u\left(h^{2}\right)=(u(h))^{2}$ where $g(x)=\sin x, h(x)=\sin (x+.01)$ on $[0, \pi]$. Then $u$ is fixed on $C[0, \pi]$.

We omit the proof. 


\section{References}

[C] S. Cater, A nonlinear generalization of a theorem on function algebras, Amer. Math. Monthly 74 (1967), no. 6, 682-685.

[GJ] L. Gillman and M. Jerison, Rings of continuous functions, D. van Nostrand, New York, 1960.

[K] J. L. Kelley, General Topology, D. van Nostrand, New York, 1955. 